\title{
Scaling of the hydrostatic skeleton in the earthworm Lumbricus terrestris
}

\author{
Jessica A. Kurth* and William M. Kier
}

\begin{abstract}
The structural and functional consequences of changes in size or scale have been well studied in animals with rigid skeletons, but relatively little is known about scale effects in animals with hydrostatic skeletons. We used glycol methacrylate histology and microscopy to examine the scaling of mechanically important morphological features of the earthworm Lumbricus terrestris over an ontogenetic size range from 0.03 to $12.89 \mathrm{~g}$. We found that $L$. terrestris becomes disproportionately longer and thinner as it grows. This increase in the length to diameter ratio with size means that, when normalized for mass, adult worms gain $\sim 117 \%$ mechanical advantage during radial expansion, compared with hatchling worms. We also found that the cross-sectional area of the longitudinal musculature scales as body mass to the $\sim 0.6$ power across segments, which is significantly lower than the 0.66 power predicted by isometry. The cross-sectional area of the circular musculature, however, scales as body mass to the $\sim 0.8$ power across segments, which is significantly higher than predicted by isometry. By modeling the interaction of muscle crosssectional area and mechanical advantage, we calculate that the force output generated during both circular and longitudinal muscle contraction scales near isometry. We hypothesize that the allometric scaling of earthworms may reflect changes in soil properties and burrowing mechanics with size.
\end{abstract}

KEY WORDS: Scaling, Allometry, Ontogeny, Annelid, Burrowing

\section{INTRODUCTION}

Body size plays a pivotal role in the structure and function of all organisms. Size affects how an organism interacts with its environment as well as the processes needed for survival (Vogel, 1988). Size also imposes physical constraints on organisms, with fundamental effects on organismal design (Schmidt-Nielsen, 1997). A range of important traits change as a function of body size, including: geometry, metabolic rate, kinematics, mechanics and even lifespan. As a consequence, almost every facet of an organism's life may be influenced by its size, including its physiology, morphology, ecology and biomechanics (Schmidt-Nielsen, 1984; Quillin, 1999; Vogel, 2013; Biewener, 2005; Hill et al., 2012). Scaling, the changes in form and function due body size, has been studied primarily in the vertebrates and in some arthropods (e.g. Schmidt-Nielsen, 1997; Biewener, 2005; Nudds, 2007; Chi and Roth, 2010). The effects of scaling on soft-bodied animals have, however, received relatively little attention. The aim of this study was to use histological and microscopic techniques to examine the effects of size and scale on components of the hydrostatic skeleton of an iconic soft-bodied animal, the earthworm.

Department of Biology, University of North Carolina, Chapel Hill, NC 27599, USA.

*Author for correspondence (jkurth@live.unc.edu)

Received 15 October 2013; Accepted 11 February 2014
Many soft-bodied organisms or parts of organisms (e.g. terrestrial and marine worms, cnidarians, echinoderms, bivalves, gastropods and nematodes) possess a hydrostatic skeleton. Hydrostatic skeletons are characterized by a liquid-filled internal cavity surrounded by a muscular body wall (Kier, 2012). Because liquids resist changes in volume, muscular contraction does not significantly compress the fluid, and the resulting increase in internal pressure allows for support, muscular antagonism, mechanical amplification and force transmission (Chapman, 1950; Chapman, 1958; Alexander, 1995; Kier, 2012).

Animals supported by hydrostatic skeletons range in size from a few millimeters (e.g. nematodes) to several meters in length (e.g. earthworms), yet little is known about scale effects on their form and function. Indeed, many individual cephalopods, which rely on a type of hydrostatic skeleton termed a muscular hydrostat, may grow through this entire size range and larger. In addition, many of these animals burrow, and the scaling of burrowing mechanics is also poorly understood compared with other forms of locomotion. We also know little about the effects of the physical properties of the soil on burrowing organisms, or how changes in body size impact soil-animal interactions. Further, this work is of interest because these animals are taxonomically diverse, they live in many environments, and are ecologically and economically important in bioturbation, ecosystem engineering and soil maintenance. Humaninduced changes in soil properties from chemicals and heavy machinery could impose size-dependent effects on burrowers that can only be predicted by understanding the scaling of the morphology and mechanics of burrowers. Finally, this research may provide insights useful for the design of biomimetic soft robots for surface locomotion and for burrowing (e.g. Trimmer, 2008; Trivedi et al., 2008; Daltorio et al., 2013).

Previous research on scaling in soft-bodied animals has provided a foundation for our understanding of the scaling of hydrostatic skeletons (Piearce, 1983; Quillin, 1998; Quillin, 1999; Quillin, 2000; Che and Dorgan, 2010; Lin et al., 2011). A number of important issues remain unexplored, however. Prior studies did not sample the smallest specimens in the size range, and were unable to measure several mechanically relevant aspects of the morphology (e.g. circular muscle cross-sectional area) (Quillin, 1998; Quillin, 2000). The results of several previous studies were also contradictory. Some experiments indicate that the hydrostatic skeleton maintains geometric and kinematic similarity with change in body size (e.g. Quillin, 1998; Quillin, 1999), while others suggest disproportionate scaling in both shape and force production (e.g. Piearce, 1983; Quillin, 2000). In addition, many hypotheses on the scaling of the hydrostatic skeleton have not yet been tested, including possible size-dependent changes in muscle stress, muscle cross-sectional area, skeletal leverage, burrowing kinematics, respiration and soil properties (Piearce, 1983; Quillin, 2000).

In this study, we investigated the scaling of functionally relevant aspects of hydrostatic skeleton morphology, using an ontogenetic 


\begin{tabular}{|ll|}
\hline List of symbols and abbreviations \\
$A$ & muscle cross-sectional area \\
$b$ & scaling exponent \\
$b_{\mathrm{o}}$ & isometric scaling exponent \\
$a_{\mathrm{mech}}$ & mechanical advantage \\
$C$ & projected coelomic area \\
$D$ & diameter \\
$L$ & body length \\
$M$ & body mass \\
$\mathrm{OLS}$ & ordinary least squares \\
$P_{\mathrm{m}}$ & pressure due to muscle contraction \\
$\mathrm{RMA}$ & reduced major axis \\
$V$ & volume \\
$\sigma_{\mathrm{m}}$ & isometric muscle stress \\
\hline
\end{tabular}

size range of the earthworm Lumbricus terrestris Linnaeus 1758. The results provide new insights into the effects of scale on hydrostatic skeletons and allow us to make testable predictions about the implications of body size for distance and mechanical advantage, force output and internal pressure production.

\section{The hydrostatic skeleton of Lumbricus terrestris}

Earthworms have a segmented hydrostatic skeleton. In L. terrestris, the number of segments remains constant during development (Piearce, 1983; Quillin, 1998). Each segment contains coelomic fluid that is largely isolated from the fluid of adjacent segments by muscular septae, allowing segments to act as essentially independent hydraulic units (Seymour, 1969). Two orientations of muscle fibres, circular and longitudinal, are present. The circular fibres act to radially thin the worm and elongate it, while the longitudinal fibres shorten the worm and cause radial expansion. Earthworms crawl and burrow using alternating waves of circular muscle and longitudinal muscle contraction that pass from anterior to posterior down the length of the body and typically involve $\sim 30$ segments (Gray and Lissman, 1938; Sims and Gerard, 1985). When the circular muscles contract, the segments thin and are thrust forward, excavating a new burrow in the soil. Contraction of the longitudinal muscles expands the segments radially, enlarging the burrow, anchoring the worm, and pulling the more posterior segments forward. There are typically one to two simultaneous waves of circular and longitudinal muscle contraction along the length of the worm during locomotion (Gray and Lissman, 1938; Quillin, 1999).

\section{Scaling of functionally relevant morphological features}

Rather than maintaining similar relative proportions with change in body size, termed isometric growth, many animals show allometric growth, in which the relative proportions change with body size (Huxley and Tessier, 1936; Schmidt-Nielsen, 1997). Allometry is common in animals with rigid skeletons, which must increase disproportionately in relative cross-section to avoid buckling due to an increase in mass. Hydrostatic skeletons lack rigid elements loaded in compression and have been hypothesized to scale isometrically (Quillin, 1998). Thus our null hypothesis is isometric scaling, which can be tested as follows. Because the density of an animal does not change with size, the mass $(M)$ is proportional to the volume $(V)$. If an earthworm scales isometrically, linear dimensions such as length $(L)$ or diameter $(D)$ are predicted to scale to the animal's $V^{1 / 3}$ and thus $M^{1 / 3}$, and any area, such as surface area or muscle cross-sectional area, will scale as $V^{2 / 3}$ and thus $M^{2 / 3}$ (see Table 1 for terms).

Alternatively, we hypothesize that the hydrostatic skeleton may scale allometrically in response to selective pressures and constraints on the animal as it changes in size. Such factors are potentially
Table 1. Definition of variables used and their isometric scaling exponents

\begin{tabular}{lll}
\hline Symbol & Variable & $\begin{array}{l}\text { Isometric scaling } \\
\text { exponent }\left(b_{\mathrm{o}}\right)\end{array}$ \\
\hline$V$ & Volume & $\alpha M$ \\
$M$ & Body mass & $\alpha V$ \\
$L$ & Body length & $\alpha M^{1 / 3}$ \\
$D$ & Diameter & $\alpha M^{1 / 3}$ \\
$A$ & Cross-sectional muscle area & $\alpha M^{2 / 3}$ \\
$C$ & Projected coelomic area & $\alpha M^{2 / 3}$ \\
$P_{\mathrm{m}}$ & Pressure due to muscle contraction & $\alpha M^{0}$ \\
$\sigma_{\mathrm{m}}$ & Muscle stress & $\alpha M^{0}$ \\
$F$ & Force output to environment & $\alpha M^{2 / 3}$ \\
\hline & &
\end{tabular}

diverse and include, for example, burrowing mechanics, internal hydrostatic pressure, respiration, heat exchange, evaporation, predation, competition and fecundity.

\section{Scaling of linear dimensions}

The scaling of the linear dimensions and muscle cross-sectional areas have important implications for the mechanics of the organism, including its kinematics, force production, mechanical advantage and internal coelomic pressure. For hydrostatic skeletons, a change in the length-to-diameter $(L / D)$ ratio affects the leverage provided by the skeletal support system. This can be understood by first referring to animals with rigid skeletons in which levers may amplify force (force output $>$ force input from muscle contraction and thus positive mechanical advantage) or amplify distance (distance output $>$ distance input from muscle contraction and thus positive distance advantage) (Kier and Smith, 1985; Vogel, 1988). Mechanical advantage and distance advantage are reciprocal. Although cylindrical hydrostatic skeletons lack rigid levers, they still allow mechanical advantage or distance advantage, depending on the orientation of the musculature in question and the $L / D$ ratio of the body (Kier and Smith, 1985). For instance, if two cylindrical bodies have identical volume, but one is more elongate and thus has a larger $L / D$ ratio, the body with the larger $L / D$ ratio will show greater distance advantage during elongation (due to shortening by circular muscle) and greater mechanical advantage during lateral expansion (due to shortening by the longitudinal muscle) compared with a hydrostatic skeleton with a smaller $L / D$ ratio (Fig. 1) (Vogel, 2013).

If $L$. terrestris scales isometrically, the $L / D$ ratio will not change with size because both $L$ and $D$ are linear dimensions and should scale as $M^{1 / 3}$. Allometry in the overall dimensions of $L$. terrestris, however, could affect the relative force and displacement of the musculature during growth. For instance, an increase in the $L / D$ ratio during growth would mean that for a given relative shortening of the circular muscle fibres, the elongation of a large worm would be relatively greater (an increase in distance advantage for the circular muscle). From the standpoint of the longitudinal muscles, an increase in the $L / D$ ratio would result in an increase in mechanical advantage of this musculature in radial expansion of the worm. Because mechanical advantage and distance advantage are reciprocal, an increase in the $L / D$ ratio would decrease the mechanical advantage of the circular musculature and decrease the distance advantage of the longitudinal musculature.

\section{Scaling of muscle cross-sectional areas and force output}

The scaling of muscle physiological cross-sectional area $(A)$ determines how relative force production by the musculature changes with size, because force due to muscle contraction is 


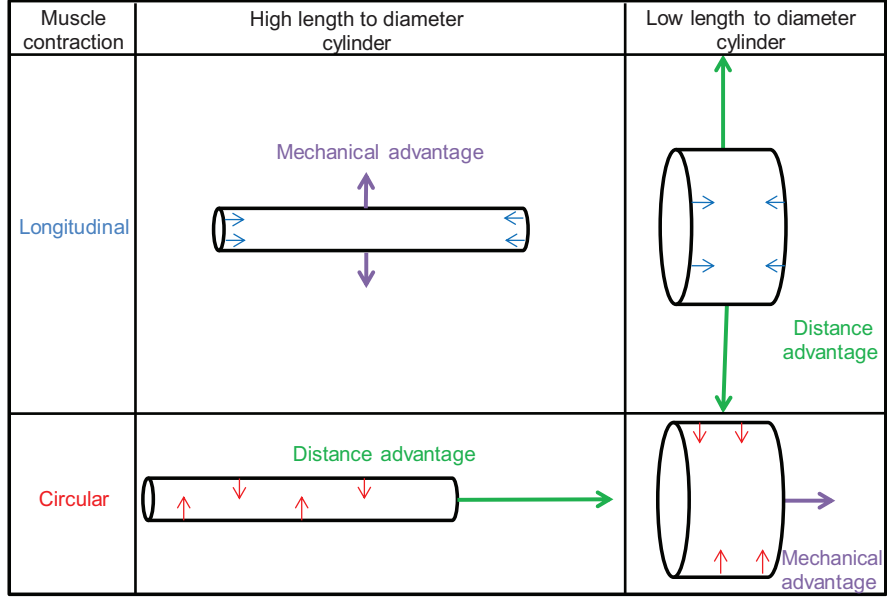

Fig. 1. Schematic comparing skeletal leverage between a high length to diameter cylinder and a low length to diameter cylinder.

proportional to cross-sectional area. If the circular and longitudinal musculature scale isometrically, the cross-sectional area of each will be proportional to $M^{2 / 3}$. The final force output the animal exerts, however, depends not only on the force-producing muscles, but also on the force-transmitting skeleton.

The force transmitted by the skeleton to the environment is a product both of the force generated by the muscles and the mechanical advantage produced by the skeleton itself:

$$
F \propto A\left(a_{\text {mech }}\right),
$$

where $F$ is the force output to the environment, $A$ is the muscle crosssectional area and $a_{\text {mech }}$ is the mechanical advantage from the skeleton. As stated above, mechanical advantage in hydrostatic skeletons will remain constant unless the $L / D$ ratio of the animal changes. If $L$. terrestris grows isometrically and thereby maintains a constant $L / D$ ratio, the mechanical advantage of the two muscle groups will not change and thus the final force output would also scale as $M^{2 / 3}$.

\section{Scaling of coelomic pressure}

The internal pressure due to muscle contraction is a function of the stress in the muscles, the cross-sectional areas of the muscles and the projected coelomic area over which the muscles act [eqn 3 from Quillin (Quillin, 1998)]:

$$
P_{\mathrm{m}}=\left(\sigma_{\mathrm{m}} A\right) C^{-1},
$$

where $P_{\mathrm{m}}$ is the pressure in the coelomic fluid due to muscle contraction, $\sigma_{\mathrm{m}}$ is the muscle stress and $C$ is the area of the coelom.
If $L$. terrestris grows isometrically and the peak isometric stress in the muscle $\left(\sigma_{\mathrm{m}}\right)$ remains constant with body size, $P_{\mathrm{m}}$ will be constant because the ratio of $A$ to $C$ would be unchanged. If the worm scales allometrically in either $A$ or $C$, then pressure will change with body size.

\section{RESULTS}

\section{Scaling of linear dimensions}

We found that both body length and diameter across all measured segments scaled allometrically (Fig. 2, Table 2). While body length scaled significantly greater $(b=0.39)$ than predicted for isometry $\left(b_{0}=0.33\right)$, the diameter of all measured segments scaled less than predicted $(b=0.292,0.278$ and 0.283 for the anterior, middle and posterior segments, respectively). As a consequence, the $L / D$ ratio increases with body size $(b=0.119,0.138$ and 0.140 for the anterior, middle and posterior segments, respectively; supplementary material Table S1) instead of remaining constant with body size as would be the case for isometry $\left(b_{0}=0.00\right)$. The number of segments active in each peristaltic wave during crawling was independent of body size (supplementary material Table S2).

\section{Scaling of muscle cross-sectional area}

The cross-sectional area of the longitudinal musculature (Fig. 3A, Table 3$)$ scaled lower than expected $(b=0.620,0.553$ and 0.591 for the anterior, middle and posterior segments, respectively) compared with isometry $\left(b_{0}=0.667\right)$ for all segments measured. However, the circular muscle cross-sectional areas of the middle and posterior segments exhibited the opposite trend. The circular muscle crosssectional area in the middle and posterior segments (Fig. 3B, Table 3 ) scaled greater than expected $(b=0.815,0.840$ for middle and posterior segments, respectively) compared with isometric scaling $\left(b_{0}=0.667\right)$. Circular muscle cross-sectional area in the anterior segment did not scale significantly differently from isometry $(b=0.690)$.

\section{Scaling of leverage and force production}

Because of the increase in the $L / D$ ratio with size, the mechanical advantage and distance advantage of the musculature changes with size (Fig. 4). We calculated that the mechanical advantage of the circular musculature will decrease with body size $(b=0.112)$ but that of the longitudinal musculature will increase $(b=0.112)$. Because the force output is proportional to the product of the mechanical advantage and cross-sectional area of the musculature, we calculated that the force output (Table 4) from the longitudinal muscle scales greater than isometry in the anterior segments $(b=0.724)$ and near isometry for the middle and posterior segments $(b=0.653$ and 0.680 for the middle and posterior segments, respectively). Force output
A

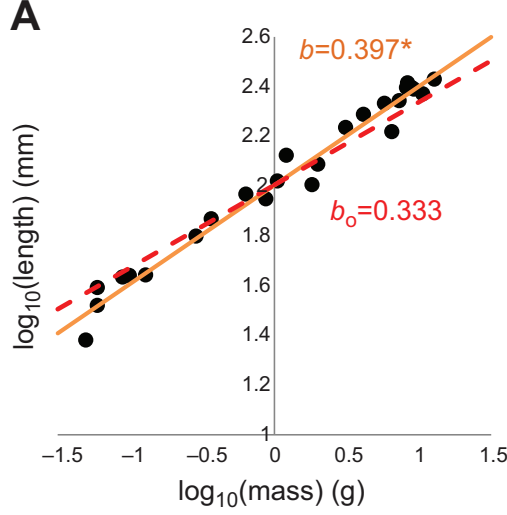

B

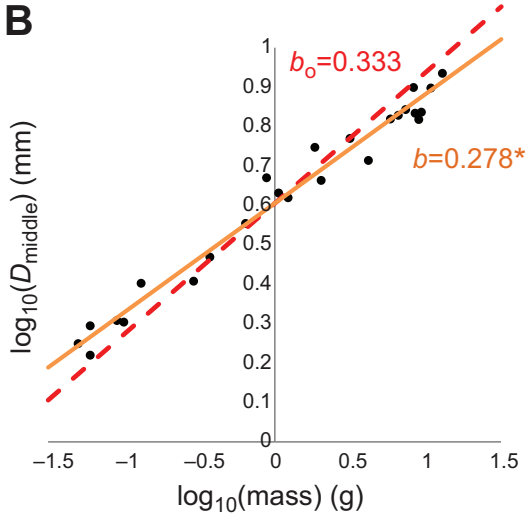

Fig. 2. Scaling of linear dimensions. (A) Log-transformed graph comparing body length with body mass. (B) Logtransformed graph comparing $D_{\text {middle }}$ (diameter of segment 30 , from the anterior) with body mass. Regressions depict the isometric scaling exponent $\left(b_{0}\right.$, dashed red line) and the scaling exponent fit to empirical data using reduced major axis regression ( $b$, solid line). $N=25$. 
Table 2. Hypothesis testing of linear dimensions using 95\% confidence intervals (Cl)

\begin{tabular}{|c|c|c|c|c|c|}
\hline Linear dimension $(y)$ & Isometric scaling exponent $\left(b_{\circ}\right)$ & RMA scaling exponent $(b)$ & Lower $95 \% \mathrm{Cl}$ & Upper $95 \%$ Cl & $R^{2}$ \\
\hline$L$ & 0.333 & $0.397^{*}$ & 0.373 & 0.423 & 0.978 \\
\hline$D_{\text {anterior }}$ & 0.333 & $0.292^{*}$ & 0.276 & 0.309 & 0.983 \\
\hline$D_{\text {middle }}$ & 0.333 & $0.278^{*}$ & 0.261 & 0.296 & 0.978 \\
\hline$D_{\text {posterior }}$ & 0.333 & $0.283^{*}$ & 0.257 & 0.312 & 0.952 \\
\hline
\end{tabular}

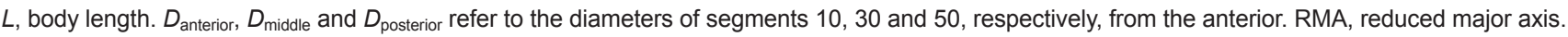

Asterisks indicate that the Cls do not overlap with $b_{0} . N=25$.

from the circular muscle of the anterior segments scales less than expected $(b=0.561)$ for isometry, but the force output of the circular muscle of the middle and posterior segments scales near isometry ( $b=0.687$ and 0.696 for the middle and posterior segments, respectively).

\section{Scaling of pressure}

We did not observe a difference from isometry $(b=0.00)$ in the ratio of the areas of the muscle and coelom $(A / C)$ for the anterior and middle segments for both the longitudinal muscles $(b=0.021$ and 0.060 for the anterior and middle segments, respectively) and the circular muscles ( $b=0.044$ and 0.049 for anterior and middle segments, respectively; Fig. 5, Table 5). The posterior segments, however, showed significant differences in the ratio of $A / C$ with body size for both the longitudinal $(b=0.146)$ and circular $(b=0.378)$ muscle.

\section{DISCUSSION}

\section{Scaling trends}

Although previous work (Quillin, 1998) had suggested that scaling of the hydrostatic skeleton should be isometric, our results show that a number of mechanically important dimensions of $L$. terrestris grow allometrically. We suspect that these differences reflect the methods used. Quillin (Quillin, 1998) used frozen sections, which tend to be subject to much greater distortion and artefact and are significantly thicker than the sections we obtained using glycol methacrylate embedding. Glycol methacrylate embedding procedures have the advantage of causing very little distortion and shrinkage, compared with other histological methods, and thinner sections allow better resolution of detail. In addition, her sections were unstained, which makes identification of the components of the tissues challenging, in particular in the smallest specimens. Instead, we employed selective stains that allowed clear differentiation of muscle and connective tissues. Finally, we used serial sections in both sagittal and transverse planes, while Quillin (Quillin, 1998) sectioned in the sagittal plane only, which complicates the measurement of the cross-sectional area of the longitudinal muscle in particular.

\section{Mechanical and distance advantage}

We found that $L$. terrestris grows disproportionately long ( $L \alpha$ $\left.M^{0.397}\right)$ and thin $\left(D \alpha M^{<0.30}\right)$, and thus the length-to-diameter ratio increases with body size $\left(L / D \propto M^{0.10}\right)$. This trend was also observed by Piearce (Piearce, 1983), who measured formalin-fixed $L$. terrestris earthworms and noted an increase in the $L / D$ ratio with mass. This increase in the $L / D$ ratio impacts the mechanics of the musculature. We estimated the effect of this allometry on the scaling of distance advantage and mechanical advantage of the musculature during elongation and shortening. From the standpoint of the circular musculature that elongates the animal, adult worms ( $10 \mathrm{~g}$ body mass) have an $\sim 117 \%$ greater distance advantage compared with $0.01 \mathrm{~g}$ hatchlings. This increase in distance advantage during elongation is consistent with the observations of Quillin (Quillin, 1999), who found that L. terrestris' stride length (i.e. distance traveled during one peristaltic wave) during crawling increased allometrically with size. From the standpoint of the longitudinal musculature that shortens the animal and thereby causes radial expansion, we estimate that adults have $117 \%$ greater mechanical advantage compared with $0.01 \mathrm{~g}$ hatchlings.

\section{Force output}

Force output to the environment is a function of both the force generated by the muscles and the transmission of that force by the skeleton. In order to predict the scaling of force output, we multiplied the scaling of the muscle cross-sectional area by the scaling of mechanical advantage of the skeleton. Although the longitudinal muscle cross-sectional area increases less than predicted by isometry $\left(A_{1} \alpha M^{0.553-0.620}\right)$, it gains mechanical advantage with size $\left(a_{\text {mech }} \alpha M^{0.112}\right)$ due to the increase in $L / D$. The increase in mechanical advantage compensates for the allometric scaling of the muscle cross-sectional area, and the force output is thus nearly isometric $\left(F_{1} \propto M^{0.653-0.724}\right)$. The circular musculature shows a similar trend; in the middle and posterior segments, the circular muscle cross-sectional area increases at a rate that is greater than
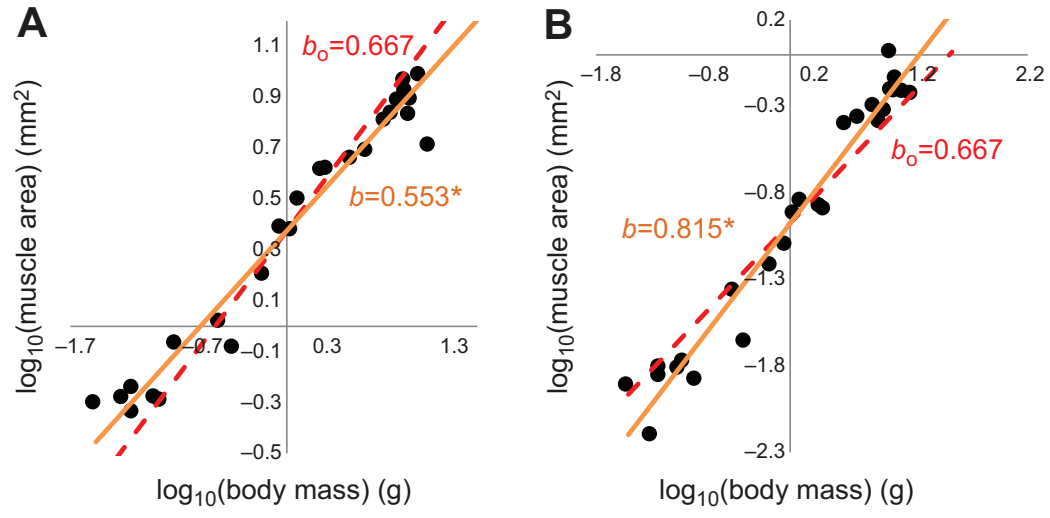

Fig. 3. Scaling of muscle cross-sectional areas. (A) Logtransformed graph of longitudinal muscle cross-sectional area in the middle segment ( $\left.A_{l, \text { middle }}\right)$ and body mass. (B) Logtransformed graph of circular muscle area in the middle segment $\left(A_{c, \text { middle }}\right)$ and body mass. Regressions depict the isometric scaling exponent ( $b_{0}$, dashed red line) and the scaling exponent fit to empirical data using reduced major axis regression ( $b$, solid line). $N=25$. 
Table 3. Hypothesis testing of muscle cross-sectional areas using $95 \% \mathrm{CI}$

\begin{tabular}{|c|c|c|c|c|c|}
\hline Muscle area $(y)$ & Isometric scaling exponent $\left(b_{\mathrm{o}}\right)$ & RMA scaling exponent $(b)$ & Lower $95 \% \mathrm{Cl}$ & Upper $95 \%$ Cl & $R^{2}$ \\
\hline$A_{\mathrm{I}, \text { anterior }}$ & 0.667 & $0.620^{*}$ & 0.580 & 0.662 & 0.976 \\
\hline$A_{l, \text { middle }}$ & 0.667 & $0.553^{*}$ & 0.508 & 0.602 & 0.967 \\
\hline$A_{\mathrm{l}, \text { posterior }}$ & 0.667 & $0.591^{*}$ & 0.535 & 0.654 & 0.948 \\
\hline$A_{\mathrm{c}, \text { anterior }}$ & 0.667 & 0.690 & 0.630 & 0.757 & 0.955 \\
\hline$A_{\mathrm{c}, \text { middle }}$ & 0.667 & $0.815^{*}$ & 0.748 & 0.888 & 0.960 \\
\hline$A_{\mathrm{C}, \text { posterior }}$ & 0.667 & $0.840^{*}$ & 0.775 & 0.909 & 0.967 \\
\hline
\end{tabular}

$A_{l}$ and $A_{c}$ are the longitudinal muscle and circular muscle cross-sectional areas, respectively. The subscripts anterior, middle and posterior denote the locations sampled. Asterisks indicate that the $\mathrm{Cls}$ do not overlap with $b_{0} . N=25$.

predicted by isometry $\left(A_{\mathrm{c}} \alpha M^{0.69-0.840}\right)$, but its mechanical advantage decreases with size $\left(a_{\text {mech }} \alpha M^{-0.112}\right)$. The force output is thus nearly isometric $\left(F_{\mathrm{c}} \propto M^{0.561-0.696}\right)$.

While our findings on the scaling of circular muscle crosssectional area are in agreement with prior research by Quillin (Quillin, 2000), our force calculations do not resolve the disproportionately low scaling of force measured by Quillin in earthworms crawling through force transducers. In the present study we were able to address several of the factors that she suggested might be responsible for the discrepancy, including scaling of muscle area and of mechanical advantage. In addition, she suggested that muscle stress might vary with body size, the kinematics of burrowing might change with size, and the resistance to soil deformation might depend on the scale of the deformation, issues that are the focus of our current investigations. An additional possibility may be the relative dimensions of the force transducers used in her experiments, which may not have measured an equivalent number of segments in the seven size classes of worms analyzed.

\section{Pressure from muscle contraction}

We found no significant trend with size of the ratio between muscle cross-sectional area and area of the coelom $\left(P_{\mathrm{m}} \alpha A / C \alpha M^{0}\right)$. Although the contractile properties of the developing muscle have not been measured, if we assume that the peak isometric stress of the muscle is independent of body size, then these results predict that the pressure produced by the musculature will be independent of body size. Internal pressure measurements of $L$. terrestris are consistent with this prediction and exhibit no trend with body size (Quillin, 1998; Keudel and Schrader, 1999).

\section{Intersegmental differences}

Several of the allometric trends differed between segments, which may reflect the relative importance of different portions of the body in burrowing. Because the peristaltic wave often dissipates as it travels down the length of the body, segments closer to the tail are likely of less importance in burrowing than those near the head (Yapp and Roots, 1956). Our data are consistent with this proposal as longitudinal force production of the anterior segments increased at a greater rate $\left(F_{1} \alpha M^{0.724}\right)$ than expected from isometry, while the middle and posterior segments scaled close to isometry. The longitudinal muscles are thought to be important in moving soil laterally to enlarge the burrow, anchor the worm and relieve stress ahead of the worm (Gray and Lissman, 1938; Keudel and Schrader, 1999).

We also found that circular muscle force production scaled disproportionately low in the anterior segments $\left(F_{\mathrm{c}} \alpha M^{0.561}\right)$, but scaled near isometry for the middle and posterior segments. The circular musculature plays an important role when the animal crawls on the surface by causing the segments to elongate and move forward. Indeed, in contrast to burrowing, the highest pressures recorded during crawling result from circular muscle contraction (Seymour, 1969). Thus, the allometric trends we observed in the anterior segments may reflect the increased importance of burrowing locomotion as L. terrestris develops, as only adult worms are found to make deep burrows.

\section{Potential selective pressures for allometric growth}

Because we identified several significant allometric growth patterns in L. terrestris, it is of interest to consider the potential selective pressures that may be acting on these animals in the environment, especially because previous research had predicted that growth would be isometric. We briefly outline below two hypotheses for the allometric trends observed. These hypotheses are not mutually exclusive and testing them is a focus of our ongoing research.

\section{Strain hardening in soil}

As an earthworm grows, selection might favor a thinner body in order to reduce 'strain hardening' during burrow formation (Piearce, 1983). Many soils, including loose granular soils and consolidated clays,
A

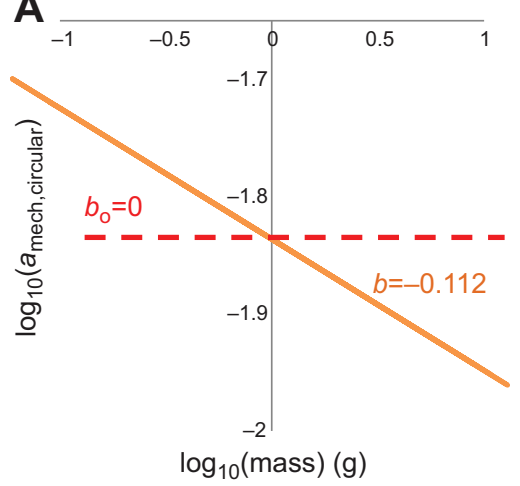

B

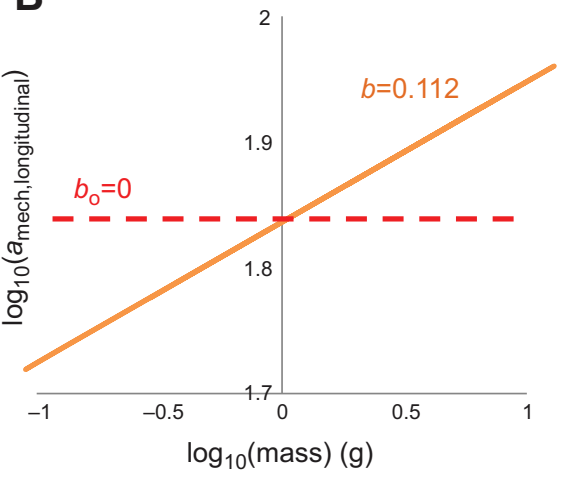

Fig. 4. Predictive model comparing mechanical advantage with body mass. (A) Mechanical advantage from circular muscle contraction ( $a_{\text {mech,circular }}$ ) and (B) mechanical advantage from longitudinal muscle contraction ( $\left.a_{\text {mech,longitudinal }}\right)$ as a function of earthworm body mass. 
Table 4. Model predicting the scaling of force output

\begin{tabular}{lll}
\hline $\begin{array}{l}\text { Modeled force } \\
\text { output }(y)\end{array}$ & $\begin{array}{l}\text { Isometric scaling } \\
\text { exponent }\left(b_{\mathrm{o}}\right)\end{array}$ & $\begin{array}{l}\text { Modeled scaling } \\
\text { exponent }(b)\end{array}$ \\
\hline$F_{\mathrm{l} \text {,anterior }}$ & 0.667 & 0.724 \\
$F_{\mathrm{l}, \text { middle }}$ & 0.667 & 0.653 \\
$F_{\mathrm{l}, \text { posterior }}$ & 0.667 & 0.680 \\
$F_{\mathrm{c}, \text { anterior }}$ & 0.667 & 0.561 \\
$F_{\mathrm{c} \text {,middle }}$ & 0.667 & 0.687 \\
$F_{\mathrm{c} \text {,posterior }}$ & 0.667 & 0.696
\end{tabular}

The RMA regression scaling exponents for each muscle cross-sectional area were multiplied with the scaling exponent of mechanical advantage.

Mechanical advantage was calculated by normalizing the changes in $L / D$ ratios with mass and calculating the reciprocal of distance advantage over $25 \%$ radial strain. $F_{\mathrm{l}}$ and $F_{\mathrm{c}}$ refer to longitudinal muscle and circular muscle force output, respectively. The subscripts anterior, middle and posterior denote the locations sampled.

exhibit this phenomenon, in which the modulus of compression or stiffness of the soil increases with increasing strain (Chen, 1975; Yong et al., 2012; Holtz et al., 2010). As an earthworm grows in crosssection, it must displace more soil radially, with a resulting increase in the stiffness of the soil surrounding the burrow. Small worms (including the hatchlings of burrowing earthworm species) are often found near the soil surface and have been hypothesized to squeeze through existing cracks and pores as 'crevice burrowers' (Arthur, 1965; Gerard, 1967). If small worms can indeed exploit these small crevices, they may avoid displacing the soil and thereby avoid the strain hardening effect. As a burrower grows and exceeds the size of the crevices, there could be a selective advantage in becoming relatively thinner to reduce this effect. This would explain why we found that $L$. terrestris grew disproportionately long and thin during ontogeny. Our results indicate that the relative reduction in diameter was achieved by reducing both the longitudinal muscle cross-section $\left(A_{1} \propto M^{0.553-0.620}\right)$ and the cross-sectional area of the coelom; the ratio of longitudinal muscle area to coelomic area did not change with body size $\left(A_{1} / C_{1} \alpha M^{0}\right)$.

\section{Crack propagation}

The increase in the length to diameter ratio we observed here may also be the result of selective pressures associated with burrowing using a mechanism termed 'crack propagation', which has been demonstrated in numerous burrowers in marine muds (Dorgan et al., 2005; Dorgan et al., 2007; Dorgan et al., 2008). Che and Dorgan (Che and Dorgan, 2010) found that small marine worms that use this mechanism, which involves lateral expansion of the anterior portion of the body to fracture the mud, are relatively thicker when burrowing and exert relatively higher forces in order to apply the required stress to propagate a crack ahead of the worm. Thus, they show allometry in body dimensions similar to that observed here for L. terrestris, with small worms being relatively thicker than large worms. Dorgan et al. (Dorgan et al., 2005), based on a review of earthworm and root growth literature, propose that terrestrial soils may fracture, but the possibility of crack propagation by terrestrial worms has not yet been investigated. This is an important area for future research because a variety of terrestrial soil environments possess mechanical properties amenable to this burrowing mechanism (Molles, 2009).

\section{Conclusions}

Our analysis indicates that, contrary to expectations from previous work, the hydrostatic skeleton of $L$. terrestris does not exhibit isometric scaling during growth. A number of functionally relevant aspects of the morphology scale allometrically, including the overall shape of the animal and the cross-sectional area of the musculature. Additional work is needed to investigate the selective pressures responsible for the increase in the $L / D$ ratio and allometry in the force production of the anterior segments. We hypothesize that changes in soil properties and burrowing mechanics with size are important. We intend to test these hypotheses and also explore the scaling of hydrostatic skeletons in other taxa, taking advantage of the taxonomic diversity and range of habitats and ecology of softbodied invertebrates. Using this approach, we hope to identify general principles of scaling in hydrostatic skeletons and burrowing mechanics.

\section{MATERIALS AND METHODS}

\section{Lumbricus terrestris collection and maintenance}

Juvenile (1-3g) worms were supplied by Knutson's Live Bait (Brooklyn, MI, USA) as well as raised from hatchlings bred in a colony maintained in the laboratory. Adult worms $(3-10 \mathrm{~g})$ were purchased locally and raised from purchased juveniles or from colony hatchlings. Hatchlings were raised from cocoons deposited by adults bred in the laboratory colony. All worms were housed in plastic bins filled with moist topsoil (composed of organic humus and peat moss) at $17^{\circ} \mathrm{C}$ (Berry and Jordan, 2001) and fed dried infant oatmeal (Burch et al., 1999).

\section{Anaesthetization, length measurements and dissection}

Each worm was anaesthetized in a $10 \%$ ethanol solution in distilled water $(\mathrm{v} / \mathrm{v})$ until quiescent, patted dry and weighed. The length was obtained after
A

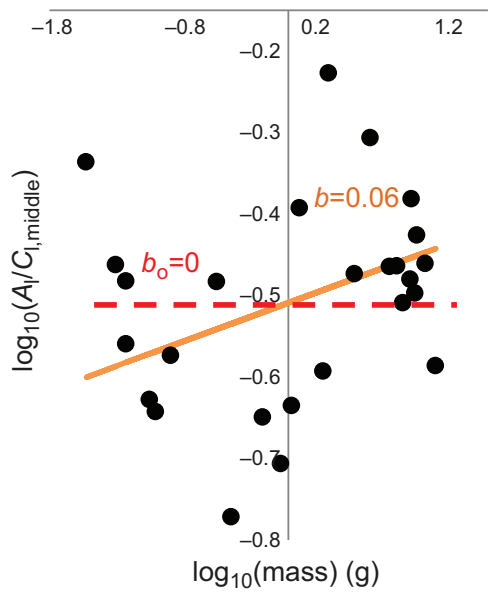

B

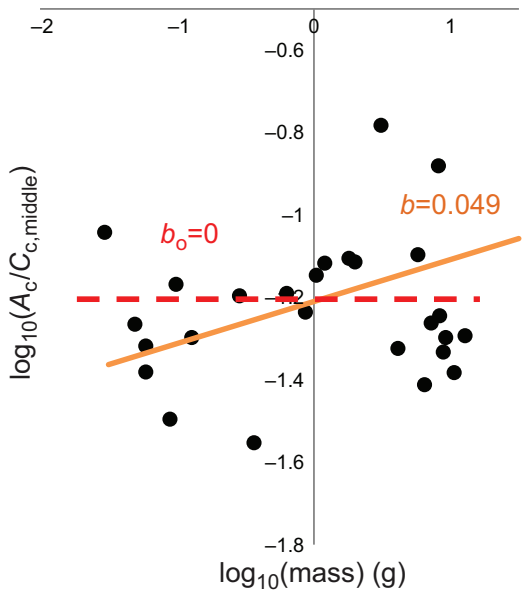

Fig. 5. Scaling of the ratio of muscle cross-sectional areas $(A)$ to projected coelomic area $(C)$ where muscle contraction is applied. The subscripts I and $\mathrm{c}$ refer to longitudinal and circular muscles, respectively. (A) Plot of

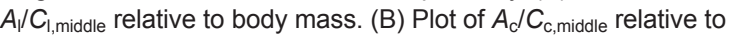
body mass. $N=25$. 
Table 5. Hypothesis testing of muscle and coelom area using $95 \% \mathrm{Cl}$

\begin{tabular}{|c|c|c|c|c|c|}
\hline Ratio $A / C(y)$ & Isometric scaling exponent $\left(b_{0}\right)$ & Measured scaling exponent $(b)$ & Lower $95 \% \mathrm{Cl}$ & Upper 95\% Cl & $R^{2}$ \\
\hline$A_{1} / C_{l, \text { anterior }}$ & 0 & 0.021 & -0.004 & 0.050 & 0.105 \\
\hline$A_{l} / C_{l, \text { middle }}$ & 0 & 0.060 & -0.043 & 0.200 & 0.055 \\
\hline$A_{\mathrm{l}} / C_{\mathrm{l}, \text { posterior }}$ & 0 & $0.146^{*}$ & 0.061 & 0.265 & 0.305 \\
\hline$A_{\mathrm{c}} / C_{\mathrm{c}, \text { middle }}$ & 0 & 0.049 & -0.100 & 0.228 & 0.020 \\
\hline$A_{\mathrm{C}} / C_{\mathrm{c}, \text { posterior }}$ & 0 & $0.378^{*}$ & 0.253 & 0.560 & 0.556 \\
\hline
\end{tabular}

$A_{l}$ and $A_{c}$ are the longitudinal muscle and circular muscle cross-sectional areas, respectively; $C_{l}$ and $C_{\mathrm{c}}$ are the projected areas of application in the coelom during longitudinal and circular muscle contraction, respectively. The subscripts anterior, middle and posterior denote the locations sampled. Asterisks indicate that the Cls do not overlap with $b_{0} . N=25$.

pulling the worm by the anterior end along the bench surface in order to straighten the body and extend the segments to a consistent resting length. Because $L$. terrestris does not add segments with growth, we measured the length of the entire body (Piearce, 1983; Quillin, 1998). The worm was then killed and three blocks of tissue containing 20 segments each were removed (segments 1-20, 21-40 and 41-60, numbering from anterior). We examined these three areas to document potential variation along the length of the worm, although particular attention was paid to segments in the anterior half of the worm because it is of greatest importance in locomotion (the posterior half of the worm is often passively dragged along) (Yapp and Root, 1956).

The tissue blocks were fixed in $10 \%$ formalin in distilled water $(\mathrm{v} / \mathrm{v})$ for 24-48 h. After fixation, the blocks were further dissected for embedding (segments 9-14, 29-34 and 49-54). We refer to segments 9-14 as 'anterior', segments 29-34 as 'middle' and segments 49-54 as 'posterior'. The anterior, middle and posterior segments were then cut in half transversely so that both transverse and sagittal sections could be obtained from each location (Fig. 6).

\section{Histology and morphometrics}

The tissue blocks were partially dehydrated in $95 \%$ ethanol and embedded in glycol methacrylate plastic (Technovit 7100, Heraeus Kulzer GmbH, Wehrheim, Germany) to minimize tissue distortion. Sections of 3-7 $\mu \mathrm{m}$ thickness were cut with a glass knife. We used a Picrosirius/Fast Green stain in order to differentiate muscle from connective tissue (López-DeLeón and Rojkind, 1985). We adapted the protocol to glycol methacrylate by staining at $60^{\circ} \mathrm{C}$ for $1-2 \mathrm{~h}$ followed by a distilled water rinse, drying and mounting of coverslips. We used Sigma Scan (Systat Software, Inc., San Jose, CA, USA) to make morphological measurements on micrographs. Longitudinal muscle cross-sectional area $\left(A_{1}\right)$, projected area of application in the coelom during longitudinal muscle contraction $\left(C_{1}\right)$ and diameter $(D)$ were measured using transverse sections. Circular muscle cross-sectional area $\left(A_{\mathrm{c}}\right)$ and projected area of application in the coelom during circular muscle contraction $\left(C_{\mathrm{c}}\right)$ were measured using sagittal sections. The earthworms prepared in this way were flattened slightly and thus had an elliptical crosssection. To determine an equivalent diameter of a circular cylinder, we used measurements of the major and minor axes to calculate the area of the ellipse and then calculated the diameter of a circle of the same area.

We estimated the scaling of internal pressure produced by muscle contraction using Eqn 2 and measurements of coelomic area from transverse and sagittal sections, with the assumption of no change with size in the peak isometric stress of the muscle $\sigma_{\mathrm{m}}$. Pressure from longitudinal muscle contraction was calculated using $A_{1}$ and $C_{1}$, while pressure from circular muscle contraction was calculated using $A_{\mathrm{c}}$ and $C_{\mathrm{c}}$ (Quillin, 1998):

$$
\begin{gathered}
P_{\mathrm{m}, \text { longitudinal }}=\left(\sigma_{\mathrm{m}} A_{1}\right) C_{1}^{-1}, \\
P_{\mathrm{m}, \text { circular }}=\left(\sigma_{\mathrm{m}} A_{\mathrm{c}}\right) C_{\mathrm{c}}^{-1} .
\end{gathered}
$$

\section{Calculation of mechanical advantage and force output}

As we describe above, the $L / D$ ratio was observed to change as a function of size and thus the mechanical advantage of the musculature changes during growth. Because the mechanical advantage is the reciprocal of the distance advantage, we calculated the mechanical advantage $\left(a_{\text {mech }}\right)$ of the circular musculature as the absolute value of the decrease in body diameter $(D)$ during circular muscle contraction divided by the resulting increase in body length $(L)$, as a function of the $L / D$ ratio, for the $25 \%$ decrease body in diameter that is typical of $L$. terrestris during movement (Quillin, 1999). Likewise, the mechanical advantage of the longitudinal muscle was calculated as the absolute value of the decrease body length of the worm
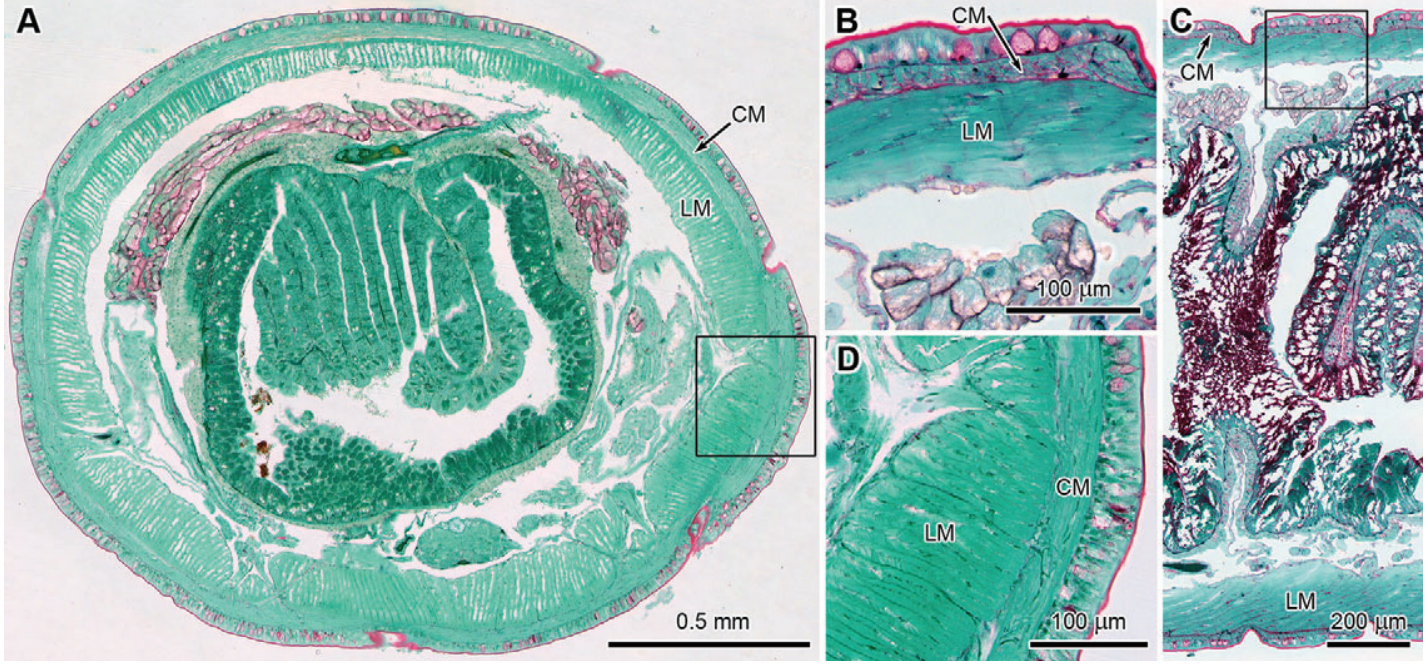

Fig. 6. Photomicrographs (brightfield microscopy) of 7- $\mu$ m-thick sections of Lumbricus terrestris stained with Picrosirius/Fast Green. (A) Transverse section through the anterior segments. (B) Inset of sagittal section shows higher magnification view of cross-section of the circular musculature. (C) Parasagittal section through the anterior segments. (D) Inset of transverse section shows higher magnification view of cross-section of the longitudinal musculature. LM, longitudinal muscle; CM, circular muscle. 
divided by the resulting increase in body diameter, as a function of the $L / D$ ratio:

$$
\begin{gathered}
a_{\text {mech,circular }}=\frac{|\Delta D|}{|\Delta L|}, \\
a_{\text {mech,longitudinal }}=\frac{|\Delta L|}{|\Delta D|} .
\end{gathered}
$$

These calculations thus provided estimates of the mechanical advantage of both the longitudinal and circular musculature as a function of size.

\section{Statistical analysis}

We used the lmodel2 package (Legendre, 2011) in R (R Development Core Team, 2013) for statistical analysis. We performed both ordinary least squares (OLS) and reduced major axis (RMA) regression on the logtransformed scaling data fit to the power function $y=a M^{b}$, where $y$ represents the morphological traits of interest, $a$ is the scaling constant, $M$ is body mass and $b$ is the scaling exponent. OLS regression does not account for error in the independent variable, while RMA regression does (Rayner, 1985). We calculated the $95 \%$ confidence intervals of the slope to determine whether the scaling exponent $b$ was significantly different from the expected isometric scaling exponent $b_{0}$, as described previously (e.g. Herrel and O'Reilly, 2006; Nudds, 2007; Chi and Roth, 2010). Both OLS regression and RMA regression fit similar scaling exponents in our analysis and were consistent in distinguishing significant differences from isometry. Because of the similarity and agreement between the models, only the RMA regressions are reported.

\section{Acknowledgements}

We thank Brina M. Montoya for her insights into soil properties as well as Tony Purdue for help with histology.

\section{Competing interests}

The authors declare no competing financial interests.

\section{Author contributions}

Both authors were involved in the planning of the experiments. J.A.K. collected and analyzed the data, and drafted the initial manuscript. W.M.K. revised the manuscript and assisted with data analysis.

\section{Funding}

This study was funded by the National Science Foundation [grant IOS-0951067 to W.M.K.].

\section{Supplementary material}

Supplementary material available online at

http://jeb.biologists.org/lookup/suppl/doi:10.1242/jeb.098137/-/DC1

\section{References}

Alexander, R. McN. (1995). Hydraulic mechanisms in locomotion. In Body Cavities: Function and Phylogeny (Selected Symposia and Monographs UZI, 8) (ed. G. Lanzavecchia, R. Valvassori and M. D. Candia Carnevali), pp 187-198. Modena: Mucchi.

Arthur, D. (1965). Form and function in the interpretation of feeding in lumbricid worms. Viewpoints Biol. 4, 204-251.

Berry, E. and Jordan, D. (2001). Temperature and soil moisture content effects on the growth of Lumbricus terrestris (Oligochaeta: Lumbricidae) under laboratory conditions. Soil Biol. Biochem. 33, 133-136.

Biewener, A. A. (2005). Biomechanical consequences of scaling. J. Exp. Biol. 208, 1665-1676.

Burch, S. W., Fitzpatrick, L. C., Goven, A. J., Venables, B. J. and Giggleman, M. A. (1999). In vitro earthworm Lumbricus terrestris coelomocyte assay for use in terrestrial toxicity identification evaluation. Bull. Environ. Contam. Toxicol. 62, 547-554

Chapman, G. (1950). Of the movement of worms. J. Exp. Biol. 27, 29-39.

Chapman, G. (1958). The hydrostatic skeleton in the invertebrates. Biol. Rev. Camb. Philos. Soc. 33, 338-371.
Che, J. and Dorgan, K. M. (2010). It's tough to be small: dependence of burrowing kinematics on body size. J. Exp. Biol. 213, 1241-1250.

Chen, W. (1975). Limit Analysis and Soil Plasticity, Developments in Geotechnical Engineering, Vol. 7. Amsterdam: Elsevier.

Chi, K. J. and Roth, V. L. (2010). Scaling and mechanics of carnivoran footpads reveal the principles of footpad design. J. R. Soc. Interface 7, 1145-1155.

Daltorio, K. A., Boxerbaum, A. S., Horchler, A. D., Shaw, K. M., Chiel, H. J. and Quinn, R. D. (2013). Efficient worm-like locomotion: slip and control of soft-bodied peristaltic robots. Bioinspir. Biomim. 8, 035003.

Dorgan, K. M., Jumars, P. A., Johnson, B., Boudreau, B. P. and Landis, E. (2005). Burrowing mechanics: burrow extension by crack propagation. Nature 433, 475-475.

Dorgan, K. M., Arwade, S. R. and Jumars, P. A. (2007). Burrowing in marine muds by crack propagation: kinematics and forces. J. Exp. Biol. 210, 4198-4212.

Dorgan, K. M., Arwade, S. R. and Jumars, P. A. (2008). Worms as wedges: effects of sediment mechanics on burrowing behavior. J. Mar. Res. 66, 219-254.

Gerard, B. (1967). Factors affecting earthworms in pastures. J. Anim. Ecol. 36, 235252

Gray, J. and Lissman, H. W. (1938). Studies in animal locomotion. VII. Locomotory reflexes in the earthworm. J. Exp. Biol. 15, 506-517.

Herrel, A. and O'Reilly, J. C. (2006). Ontogenetic scaling of bite force in lizards and turtles. Physiol. Biochem. Zool. 79, 31-42.

Hill, R. W., Wyse, G. A. and Anderson, M. (2012). Animal Physiology, 3rd edn. Sunderland, MA: Sinauer Associates.

Holtz, R. D., Kovacs, W. D. and Sheahan, T. C. (2010). An Introduction to Geotechnical Engineering, 2nd edn. Upper Saddle River, NJ: Prentice Hall.

Huxley, J. S. and Tessier, G. (1936). Terminology of relative growth. Nature 137, 780781

Keudel, M. and Schrader, S. (1999). Axial and radial pressure exerted by earthworms of different ecological groups. Biol. Fertil. Soils 29, 262-269.

Kier, W. M. (2012). The diversity of hydrostatic skeletons. J. Exp. Biol. 215, 1247-1257. Kier, W. M. and Smith, K. K. (1985). Tongues, tentacles and trunks: the biomechanics of movement in muscular-hydrostats. Zool. J. Linn. Soc. 83, 307-324.

Legendre, P. (2011). Imodel2: Model II Regression. $R$ Package Version 1.7-0. Available at $h$ ttp://CRAN.R-project.org/package=Imodel 2 .

Lin, H. T., Slate, D. J., Paetsch, C. R., Dorfmann, A. L. and Trimmer, B. A. (2011). Scaling of caterpillar body properties and its biomechanical implications for the use of a hydrostatic skeleton. J. Exp. Biol. 214, 1194-1204.

López-De León, A. and Rojkind, M. (1985). A simple micromethod for collagen and total protein determination in formalin-fixed paraffin-embedded sections. J. Histochem. Cytochem. 33, 737-743.

Molles, M. C. (2009). Ecology: Concepts \& Applications, 5th edn. New York, NY: McGraw-Hill.

Nudds, R. L. (2007). Wing-bone length allometry in birds. J. Avian Biol. 38, 515-519.

Piearce, T. G. (1983). Functional morphology of lumbricid earthworms, with special reference to locomotion. J. Nat. Hist. 17, 95-111.

Quillin, K. J. (1998). Ontogenetic scaling of hydrostatic skeletons: geometric, static stress and dynamic stress scaling of the earthworm lumbricus terrestris. J. Exp. Biol. 201, 1871-1883.

Quillin, K. J. (1999). Kinematic scaling of locomotion by hydrostatic animals: ontogeny of peristaltic crawling by the earthworm lumbricus terrestris. J. Exp. Biol. 202, 661674

Quillin, K. J. (2000). Ontogenetic scaling of burrowing forces in the earthworm Lumbricus terrestris. J. Exp. Biol. 203, 2757-2770.

R Development Core Team (2013). R: A Language and Environment for Statistical Computing. Vienna, Austria: R Foundation for Statistical Computing.

Rayner, J. M. V. (1985). Linear relations in biomechanics: the statistics of scaling functions. J. Zool. 206, 415-439.

Schmidt-Nielsen, K. (1984). Scaling Why is Animal Size So Important? Cambridge: Cambridge University Press.

Schmidt-Nielsen, K. (1997). Animal Physiology: Adaptation and Environment, 5th edn. Cambridge: Cambridge University Press.

Seymour, M. K. (1969). Locomotion and coelomic pressure in Lumbricus terrestris. J. Exp. Biol. 51, 47-58.

Sims, R. W. and Gerard, B. M. (1985). Earthworms: Keys and Notes for the Identification and Study of the Species. London: Brill Archive.

Trimmer, B. A. (2008). New challenges in biorobotics: incorporating soft tissue into control systems. Appl. Bionics Biomech. 5, 119-126.

Trivedi, D., Rahn, C. D., Kier, W. M. and Walker, I. D. (2008). Soft robotics: biological inspiration, state of the art, and future research. Appl. Bionics Biomech. 5, 99-117.

Vogel, S. (1988). Life's Devices: The Physical World of Animals and Plants. Princeton, NJ: Princeton University Press.

Vogel, S. (2013). Comparative Biomechanics: Life's Physical World, 2nd edn. Princeton, NJ: Princeton University Press.

Yapp, W. B. and Roots, B. I. (1956). Locomotion of worms. Nature 177, 614-615

Yong, R. N., Nakano, M. and Pusch, R. (2012). Environmental Soil Properties and Behavior. Boca Raton, FL: CRC Press.

Chilos. Soc 33, 338-371.

\begin{abstract}
Behavor. Boca Raton, FLiCRC Press.
\end{abstract}

